\title{
Dispersão e Caracterização de Frutos de Myrceugenia euosma em Floresta Ombrófila Mista no Sul do Brasil
}

\author{
Newton Clóvis Freitas da Costa ${ }^{1}$, Lilian Iara Bet Stedille ${ }^{2}$, \\ Paula Iaschitzki Ferreira ${ }^{3}$, Juliano Pereira Gomes ${ }^{3}$, Adelar Mantovani ${ }^{2}$ \\ ${ }^{1}$ Programa de Pós-graduação em Engenharia Florestal, Universidade do Estado de Santa Catarina - \\ UDESC, Lages/SC, Brasil \\ ${ }^{2}$ Departamento de Engenharia Florestal, Universidade do Estado de Santa Catarina - UDESC, Lages/SC, Brasil \\ ${ }^{3}$ Programa de Pós-graduação em Produção Vegetal, Universidade do Estado de Santa Catarina - UDESC, Lages/SC, Brasil
}

\begin{abstract}
RESUMO
Este estudo objetivou caracterizar aspectos relacionados à dispersão de propágulos de Myrceugenia euosma (O. Berg) D. Legrand (Myrtaceae) em um fragmento de Floresta Ombrófila Mista em Urupema, SC. Para isso, foram avaliados 29 indivíduos reprodutivos quanto à frequência de visitantes, chuva de propágulos, remoção e caracterização de frutos. Registraram-se 140 visitas de pássaros, sem ocorrência de período preferencial. As espécies com maior frequência de visita e maior consumo de frutos foram Zonotrichia capensis (tico-tico) e Stephanophorus diadematus (cabeça-de-velho), consideradas potenciais dispersoras. A frequência de coletores contendo propágulos de M. euosma diminuiu com o aumento da distância, apresentando comportamento distinto em função do tipo de propágulo avaliado. Também foram verificadas sementes intactas junto às fezes das aves nos coletores. A taxa de remoção de frutos depositados no chão foi de $22 \%$, isso demonstra a relevância desse tema no processo de dispersão das espécies, merecendo atenção na realização de estudos futuros.
\end{abstract}

Palavras-chave: síndrome de dispersão, remoção de frutos, ornitocoria.

\section{Dispersal and Characteristics of Myrceugenia euosma Fruit in Mixed Ombrophilous Forests, Southern Brazil}

\begin{abstract}
This study aimed to characterize aspects related to propagules dispersion of Myrceugenia euosma (Myrtaceae) in a fragment of Mixed Ombrophillous Forest, Urupema, SC. For this, twenty-nine seed tree were evaluated for focal observation of visitors, propagules rain, removal and characterization of fruits. We recorded 140 visits of birds, occurring without preferential visit period. The species with higher visiting frequency and consumption of fruits were Zonotrichia capensis (Rufous-collared Sparrow) and Stephanophorus diadematus (Diademed Tanager), considered as potential dispersers. The frequency of collectors containing M. euosma propagules decreased with increased distance, presenting distinct behavior depending the type of propagule evaluated. We also found intact seeds in bird feces inside the collectors. The removal rate of fruits deposited on the ground was $22 \%$, demonstrating that this is an important topic on the dispersal process and deserves attention in future studies.
\end{abstract}

Keywords: dispersal syndrome, fruit removal, ornithochory. 


\section{INTRODUÇÃO}

Dispersão é um processo pelo qual as sementes são removidas das imediações da planta-mãe para distâncias consideradas "seguras" em que a predação e a competição teoricamente são menores (Cordeiro \& Howe, 2003; Howe \& Miriti, 2004). É descrita como uma adaptação para aumentar a probabilidade de sobrevivência, uma vez que os principais fatores responsáveis pela mortalidade das plântulas são a densidade e a proximidade em relação à planta-mãe (Janzen, 1970; Howe, 1993). A dispersão de sementes apresenta ligação direta com a dinâmica das florestas, sendo um dos processos mais importantes no controle e manutenção da diversidade biológica dos ecossistemas (Harper, 1977; Howe \& Smallwood, 1982; Forget, 1993). É fundamental para manter a estrutura demográfica e o padrão espacial de espécies vegetais, pois representa a ponte que une a polinização ao recrutamento que levará ao estabelecimento de novas plantas (Harper, 1977). Intervenções nesses processos podem afetar de modo irreversível o equilíbrio dinâmico das populações (Terborgh, 1986).

Em florestas tropicais é muito comum a dispersão intermediada por animais, que constitui uma forma eficaz para a realização do fluxo gênico e controle da estrutura genética das populações (Jordano \& Godoy, 2002; Saravy et al., 2006). Na Mata Atlântica, cerca de $87 \%$ das espécies arbóreas produzem frutos carnosos, porcentagem que chega a $90 \%$ em algumas áreas (Frankie et al., 1974; Fleming et al., 1987; Galetti et al., 2006; Liebsch, 2007). Myrtaceae, por ser uma das famílias botânicas de maior riqueza e abundância de arbóreas nesse bioma (Mauhs \& Backes, 2002; Sobral, 2003; Sonego et al., 2007), possui um grande número de espécies atrativas para a fauna (Gressler et al., 2006). No Brasil, os principais dispersores de Myrtaceae são aves e macacos, porém outros mamíferos, répteis, peixes e formigas interagem de forma eventual, podendo contribuir para a dispersão de sementes (Pizo, 2003; Gressler et al., 2006). Contudo, a fragmentação de hábitats, na Mata Atlântica, tem causado um declínio de frugívoros, resultando em um empobrecimento da fauna dispersora (Silva \& Tabarelli, 2000; Pizo, 2004; Hansen \& Galetti, 2009).

Nesse contexto, estudos de dispersão de frutos podem fornecer informações importantes, que contribuem para a compreensão das interações ecológicas dos ecossistemas e hábitats e podem auxiliar nos processos de conservação da biodiversidade (Galetti et al., 2006). No entanto, poucos trabalhos relacionados à ecologia da dispersão têm sido desenvolvidos para espécies de Myrtaceae no Brasil (e.g., Pizo, 2003; Gressler et al., 2006; Côrtes et al., 2009; Silva et al., 2013; este estudo). Considera-se que além da conservação da biodiversidade esses estudos possibilitam apontar potenciais de uso das espécies, seja na restauração de ecossistemas, seja na arborização ou em processos de domesticação de plantas.

Dessa forma, objetivou-se caracterizar aspectos relacionados à dispersão de propágulos de Myrceugenia euosma em um fragmento de Floresta Ombrófila Mista. Para isso, avaliaram-se algumas questões referentes a: I) existência de possíveis dispersores para a espécie; II) frequência de ocorrência de propágulos em relação ao aumento da distância da planta matriz; III) remoção de frutos depositados no chão em ambientes com diferentes estágios de sucessão; e IV) caracterização dos frutos da espécie.

\section{MATERIAIS E MÉTODOS}

\subsection{Espécie estudada}

Myrceugenia euosma (O. Berg) D. Legrand (Myrtaceae), comumente conhecida por guamirim, é endêmica do domínio da Floresta Atlântica e apresenta uma distribuição geográfica abrangendo parte do Sudeste e do Sul do Brasil. Ocorre na Floresta Ombrófila Mista (FOM), em Floresta Nebular, nas margens de cursos d'água (Sobral, 2003) e apresenta seletividade higrófita e heliófila (Legrand \& Klein, 1970). A espécie possui hábito de arbustivo a árvore, com cerca de 3-8 $\mathrm{m}$ de altura, $25 \mathrm{~cm}$ de diâmetro e raio da copa com cerca de 2,5-3 m. Apresenta flores brancas, axilares (uma a duas por axila), com pedicelos de 7-10 mm, podendo ser observadas de outubro a janeiro no Estado do Rio Grande do Sul (Sobral, 2003) e de setembro a fevereiro no Estado de Santa Catarina (Legrand \& Klein, 1970). Os frutos são do tipo baga, globosos, com até $10 \mathrm{~mm}$ de diâmetro, negros quando maduros e com frutificação ocorrendo de julho a outubro (Sobral, 2003). Apesar disso, frutos maduros foram registrados pelo presente estudo nos meses de janeiro e fevereiro. Aspectos da ecologia da polinização e dispersão não são citados na literatura. 


\section{2. Área de estudo}

Este estudo foi conduzido em fevereiro de 2012, na Fazenda das Nascentes (27052'37,33"S e 4955'14,39”'W), localizada no município de Urupema, no Planalto Serrano do Estado de Santa Catarina. A área da fazenda pertence a uma RPPN (Reserva Particular do Patrimônio Natural) de propriedade da Klabin SA (Figura 1I). É conhecida por conter quatro das cinco nascentes do Rio Caveiras, responsável pelo abastecimento da cidade de Lages e afluente do Rio Canoas. Possui uma área de aproximadamente 1.350 ha, com altitude média de $1.435 \mathrm{~m}$, apresentando Áreas de Alto Valor de Conservação (Martins-Ramos et al., 2011). O relevo da região é composto pelas unidades do Planalto de
Lages, das Planícies Fluviais e da Serra Geral, com solos predominantes de tipo Nitossolos Háplicos e Cambissolos Húmicos, originados a partir de rochas basálticas (EMBRAPA, 1999).

Segundo Martins-Ramos et al. (2011), aproximadamente 85\% da extensão da fazenda são recobertos por Floresta Ombrófila Mista Altomontana, sendo Myrceugenia euosma a espécie arbórea de maior ocorrência, seguida por Vernonanthura discolor (Spreng.) H. Rob., Cinnamomum amoenum (Nees) Kosterm., Ocotea pulchella (Nees e Mart.) Mez, Persea willdenovii Kosterm., Araucaria angustifolia (Bert.) O. Kuntze, Drimys angustifolia Miers, Myrsine coriacea (Sw.) R. Br. ex Roem. e Schult., Podocarpus lambertii Klotzsch ex Endl. e Prunus myrtifolia (L.) Urb. A vegetação se
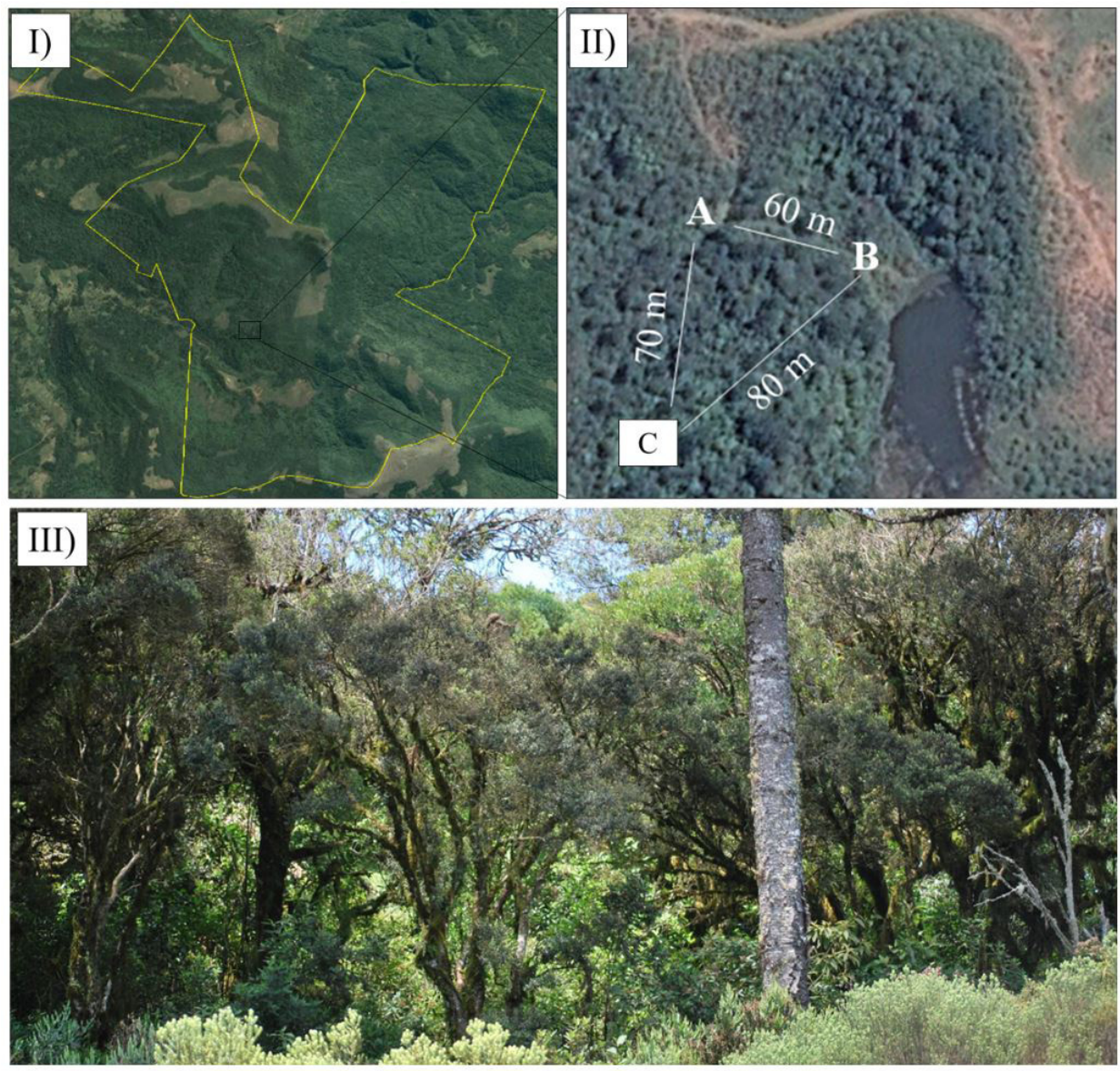

Figura 1. Local do estudo de dispersão de Myrceugenia euosma, em Urupema, SC. I) Fazenda das Nascentes; II) Localização e posicionamento dos grupos focais; III) Grupo focal C, como exemplificação, composto por seis indivíduos.

Figure 1. Study site of dispersion of Myrceugenia euosma, Urupema, SC. I) Fazenda das Nascentes; II) Location and positioning of the focus group; III) Focus group C, as exemplification, composed by six individuals. 
intercala entre áreas úmidas, frequentes nas partes mais baixas da fazenda, e campos naturais, os quais eram utilizados principalmente para pastagem de bovinos.

O clima da região é mesotérmico subtropical úmido, do tipo Cfb Köppen-Geiger, com verões frescos, sem estação seca, com geadas severas frequentes, temperatura média dos meses mais quentes inferiores a $22{ }^{\circ} \mathrm{C}$ e precipitação anual variando em torno de 1.600 a $1.900 \mathrm{~mm}$ (Alvares et al., 2014). Os dados climáticos locais, para o mês de realização do estudo (fevereiro de 2012), apresentaram temperatura média de $18,7^{\circ} \mathrm{C}$, com $160,8 \mathrm{~mm}$ de precipitação e umidade relativa do ar média de $83 \%$. Esses valores foram obtidos da estação meteorológica EPAGRI/CIRAM de Urupema, localizada a $1.800 \mathrm{~m}$ do local de realização do estudo.

\subsection{Amostragem e coleta de dados}

Para o estudo da dispersão de Myrceugenia euosma, 29 indivíduos reprodutivos foram avaliados quanto à: a) presença de visitantes; b) chuva de propágulos em coletores; c) remoção de frutos depositados no chão, e d) caracterização de frutos. Foram considerados três tipos de propágulos, sendo estes: frutos imaturos (coloração verde), frutos maduros (coloração negra) e sementes. No período das observações, os indivíduos de M. euosma apresentaram grande produção de frutos, sendo a maioria de frutos imaturos (Figura 2III). Apesar da relativa menor proporção de frutos maduros, a quantidade foi suficiente para a realização do estudo, considerando que os eventos de dispersão podem ocorrer mesmo fora do período de pico de maturação.
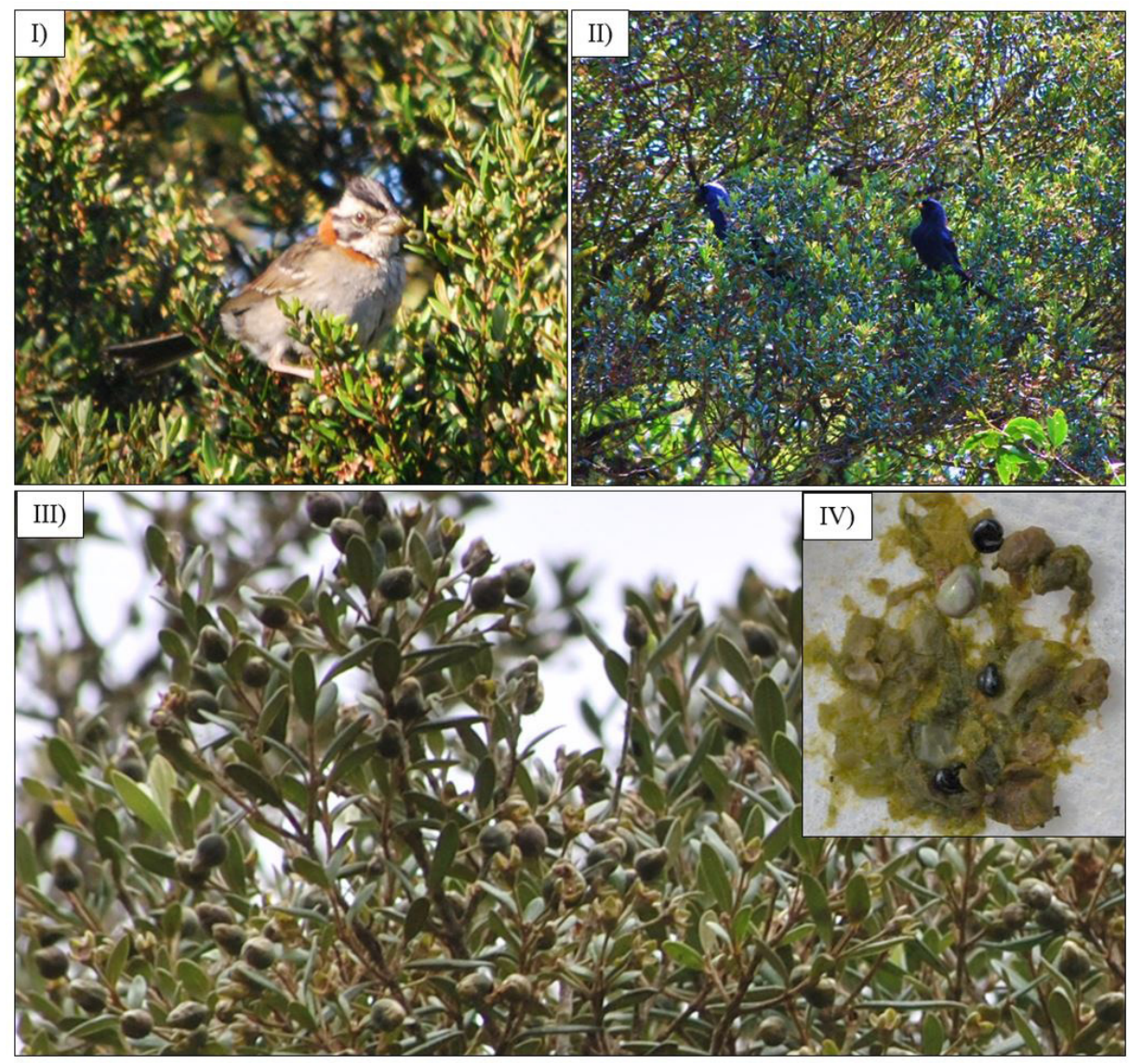

Figura 2. Registros realizados durante o estudo de dispersão de Myrceugenia euosma na Fazenda das Nascentes, Urupema, SC. I) Zonotrichia capensis; II) Stephanophorus diadematus; III) Ramos com frutos; e IV) Sementes em meio a fezes de aves encontradas dentro dos coletores.

Figure 2. Realized register during the dispersal study of Myrceugenia euosma, in the Fazenda das Nascentes, Urupema, SC. I) Zonotrichia capensis; II) Stephanophorus diadematus; III) Branch with fruits; e IV) Seeds among the feces of birds found in the collectors. 
A presença de visitantes foi avaliada pela técnica de observações focais em três grupos de indivíduos (devido à proximidade dos indivíduos dentro de cada grupo, eles foram avaliados em conjunto), a saber: 1) grupo A: quatro indivíduos, 2) grupo B: sete indivíduos, e 3) grupo C: seis indivíduos (Figura 1). Sempre que possível, foi registrado o comportamento dos visitantes em relação ao consumo efetivo de frutos, para a posterior avaliação de seu papel como potenciais agentes dispersores de M. euosma. As observações focais foram realizadas com auxílio de binóculos (8X40) e câmera digital (lente 55-200 mm), durante seis dias consecutivos, em quatro períodos do dia (08:00, 12:00, 16:00 e 18:00 h). O tempo de cada observação foi de uma hora, e cada grupo foi observado duas vezes por período (não simultâneos), totalizando $24 \mathrm{~h}$ de observação. Durante as observações foi registrado se os visitantes consumiam efetivamente os frutos ou não.

A chuva de propágulos foi avaliada em três indivíduos reprodutivos de Myrceugenia euosma, os quais foram selecionadas aleatoriamente, mantendo-se uma distância mínima de $30 \mathrm{~m}$ de outros indivíduos da espécie. A avaliação foi feita em 36 coletores de $0,5 \times 0,5 \mathrm{~m}$, instalados a 0,2 m de altura. Em cada um dos três indivíduos foram posicionados 12 coletores, três em cada quadrante, nas distâncias de 1, 3 e 5 metros a partir do tronco. Foram realizadas avaliações diárias, durante seis dias, para registrar a frequência de coletores com a presença de propágulos da espécie.

A remoção de frutos do solo (remoção secundária) foi avaliada por um experimento instalado em dois ambientes com diferentes estádios sucessionais. Ambiente I: mata fechada, em estádio de sucessão secundário, com presença de sub-bosque e dossel acima de $10 \mathrm{~m}$ de altura, caracterizada principalmente por espécies como Araucaria angustifolia, Drymis brasiliensis e Ilex paraguariensis. Ambiente II: vegetação aberta, em estádio de sucessão inicial, caracterizada principalmente por Baccharis spp. com cerca de 2,5 m de altura. Para cada ambiente foram montadas, sobre a superfície do solo, cinco parcelas de $0,5 \mathrm{~m}^{2}$, distanciadas $50 \mathrm{~m}$ entre si, contendo 100 frutos maduros cada. A avaliação da remoção foi realizada 48 h após a instalação do experimento, com a contagem direta dos frutos que foram removidos em cada parcela.
Salienta-se que durante esse experimento não ocorreu precipitação pluviométrica.

Para a caracterização de frutos de Myrceugenia euosma foram avaliadas as seguintes características: a) quantidade de frutos total em um indivíduo; b) diâmetro e peso dos frutos maduros; e c) número de sementes por fruto maduro. Para isso, frutos foram coletados aleatoriamente de oito árvores não pertencentes aos experimentos/observações anteriores. A quantidade de frutos na copa foi estimada para um indivíduo, pela contagem dos frutos presentes em um quadrante da copa, com raio médio equivalente a 2,28 m. A massa total de frutos desse indivíduo foi obtida multiplicando-se o número total de frutos pelo seu peso médio. Com auxílio de lupa de bancada e paquímetro digital, 50 frutos foram utilizados para a contagem de sementes e 57 para a medida do diâmetro do fruto. Para a pesagem utilizaram-se 1.000 frutos, pesados em lotes de 100 unidades, em uma balança semianalítica com precisão de $0,001 \mathrm{~g}$. Por meio de um caminhamento aleatório foi realizada uma avaliação das espécies arbóreas com oferta de frutos para o mesmo período e local de realização deste estudo.

\subsection{Análise dos dados}

Para a análise dos dados das observações focais foi utilizado o teste $\mathrm{H}$ de Kruskal-Wallis, afim de verificar se o número de visitas diferia entre os períodos de observação. A análise foi realizada para todas as espécies visitantes em conjunto e também individualmente para as três espécies que apresentaram maior frequência. A chuva de propágulos nos coletores foi analisada pelo teste Qui-quadrado- $\chi^{2}$ (Zar, 1984). Nesse teste foi verificado se o número de coletores contendo propágulos associa-se ao tipo de propágulo observado e também se o aumento da distância dos coletores associa-se à frequência de coletores contendo propágulos. Quanto ao experimento de remoção de frutos depositados no chão, para realizar a comparação entre os ambientes, primeiramente foi realizada a transformação logarítmica dos dados, para atingir os pressupostos da análise e, em seguida, utilizou-se o teste $t$ de student para comparação de médias. A caracterização de frutos foi avaliada por estatísticas descritivas. 


\section{RESULTADOS}

\subsection{Observação focal dos visitantes}

Foram registrados 140 eventos de visitas de aves nos indivíduos de Myrceugenia euosma, sendo identificadas nove espécies visitantes (Tabela 1 ). O número médio de visitas verificado durante as observações focais foi de 6 visitas por hora, variando de 0 (zero), no período das 12:00 h e das 18:00 h, até 20 visitas por hora no período das 16:00 h (Tabela 2). O teste de Kruskal-Wallis revelou que o número de visitas realizadas não diferiu entre os períodos observados $(\mathrm{H}=4,9 ; \mathrm{gl}=3 ; \mathrm{p}=0,18)$. $\mathrm{O}$ mesmo resultado foi observado ao se analisarem separadamente as três espécies de maior frequência relativa: Zonotrichia capensis, $(\mathrm{H}=2,8 ; \mathrm{gl}=3 ; \mathrm{p}=0,42)$, Stephanophorus diadematus $(\mathrm{H}=3,1 ; \mathrm{gl}=3 ; \mathrm{p}=0,37)$ e Contopus cinereus/Muscipipra vetula $(\mathrm{H}=1,8 ; \mathrm{gl}=3 ; \mathrm{p}=0,60)$.

Do total de 140 registros nos quais as aves pousaram sobre os indivíduos de M. euosma, em apenas 22 casos foi possível observar que ocorreu consumo efetivo de frutos, totalizando 98 frutos de M. euosma consumidos. O maior número de visitas com consumo de frutos foi de Stephanophorus diadematus (11 registros) (Figura 2II), seguido por Zonotrichia capensis (7 registros) (Figura 2I) e por, pelo menos, quatro outras espécies (Contopus cinereus/Muscipipra vetula, Sicalis flaveola, Leptasthenura striolata e uma sp. não identificada), que apresentaram apenas um registro de consumo efetivo de frutos. Nas demais visitas, apesar de algumas aves tocarem nos frutos, não foi possível visualizar consumo efetivo.

\subsection{Caracterização dos frutos}

O número de frutos contabilizados em um quadrante da copa de um indivíduo de M. euosma, com raio médio de $2,28 \mathrm{~m}$, foi de 12.800 e a estimativa para o total de frutos, de 51.200. Os frutos de M. euosma apresentaram em média 4,24 mm de diâmetro, peso de 6,83 g (para lotes contendo 100 sementes) e número de sementes de aproximadamente 3 (Tabela 3 ).

Tabela 1. Espécies de aves e respectiva frequência relativa de visitas em árvores de Myrceugenia euosma, Fazenda das Nascentes, Urupema, SC.

Table 1. Bird species and their relative frequency of visitation in Myrceugenia euosma trees, in the Fazenda das Nascentes, Urupema, SC.

\begin{tabular}{lcc}
\multicolumn{1}{c}{ Espécie } & $\mathbf{N}^{\circ}$ de visitas (frequência) & Nome comum \\
\hline $\begin{array}{l}\text { Zonotrichia capensis (Statius Muller, 1776) } \\
\text { Stephanophorus diadematus (Temminck, 1823) }\end{array}$ & $44(31 \%)$ & tico-tico \\
$\begin{array}{l}\text { Contopus cinereus (Spix, 1825) e Muscipipra vetula } \\
\text { (Lichtenstein, 1823) }\end{array}$ & $28(20 \%)$ & cabeça de velho \\
Sicalis flaveola (Linnaeus, 1766) & $20(14 \%)$ & cinzinha* $^{*}$ \\
Saltator maxillosus (Cabanis, 1851) & $10(7 \%)$ & canário da terra \\
Leptasthenura striolata (Pelzeln, 1856) & $7(5 \%)$ & trinca ferro bico grosso \\
Turdus amaurochalinus (Cabanis, 1850) & $4(3 \%)$ & grimpeiro \\
Poospiza thoracica (Nordmann, 1835) & $2(1 \%)$ & sabiá branco \\
Sem identificação & $1(1 \%)$ & peito de pinhão \\
\hline Total & $24(18 \%)$ & --- \\
\hline
\end{tabular}

*Nome comum utilizado neste estudo para duas espécies de difícil diferenciação no campo.

Tabela 2. Número de visitas de aves registradas em indivíduos de Myrceugenia euosma em quatro diferentes períodos do dia, para 24 h de observação focal. DP = desvio padrão.

Table 2. Number of bird visits registered in individuals of Myrceugenia euosma at four different times of the day, for 24 hours of focal observation. DP = standard deviation.

\begin{tabular}{ccccccccc} 
Período & \multicolumn{9}{c}{ Dia de observação } & \multicolumn{2}{c}{ Total } & $\begin{array}{c}\text { Média } \pm \\
\text { DP }\end{array}$ \\
\cline { 2 - 7 } & I & II & III & IV & V & VI & & $7,5 \pm 3,8$ \\
\hline $08: 00 \mathrm{~h}$ & 4 & 11 & 5 & 8 & 4 & 13 & 45 & $4,2 \pm 3,3$ \\
\hline $12: 00 \mathrm{~h}$ & 4 & 0 & 5 & 10 & 3 & 3 & 25 & $8,7 \pm 7,3$ \\
\hline $16: 00 \mathrm{~h}$ & 4 & 12 & 3 & 1 & 12 & 20 & 52 & $3,0 \pm 2,8$ \\
\hline $18: 00 \mathrm{~h}$ & 5 & 4 & 1 & 0 & 7 & 1 & 18 & \\
\hline
\end{tabular}


Para o mesmo período e mesmo local da realização do presente estudo, foram verificadas outras cinco espécies arbóreas com presença de frutos imaturos ou maduros, a saber: Drymis brasiliensis Miers, Ilex paraguariensis A. St. Hill, Solanum sanctaecatharinae Dunal, Solanum variabile Mart e Rhamnus sphaerosperma Sw.

\subsection{Coletores de propágulos}

Após seis dias de observação registrou-se que um total de 90 coletores continham diferentes tipos de propágulos de Myrceugenia euosma (Tabela 4). Independentemente da distância dos coletores, o teste $\chi^{2}$ revelou que não existe associação entre a frequência de coletores contendo propágulos e o tipo de propágulo observado, $\left(\chi^{2}=4,8 ; \mathrm{p} \geq 0,05\right)$. Já a frequência de coletores contendo propágulos diminuiu com o aumento da distância da planta matriz, independentemente do tipo de propágulo observado $\left(\chi^{2}=60,1 ; \mathrm{p}<0,05\right)$.

A frequência de coletores com frutos imaturos diminuiu na comparação feita de $1 \mathrm{~m}$ a $3 \mathrm{~m}$ de distância da matriz $\left(\chi^{2}=16,7 ; \mathrm{p}<0,05\right)$, porém não diferiu entre as distâncias de $3 \mathrm{~m}$ e $5 \mathrm{~m}\left(\chi^{2}=3,1 ; \mathrm{p} \geq 0,05\right)$. A frequência de coletores contendo frutos maduros, tanto na comparação de $1 \mathrm{~m}$ a $3 \mathrm{~m}\left(\chi^{2}=16,4 ; \mathrm{p}<0,05\right)$, quanto de $3 \mathrm{~m}$ a $5 \mathrm{~m}\left(\chi^{2}=5,1 ; \mathrm{p}<0,05\right)$ diminuiu com o aumento da distância. Já para as sementes a frequência de coletores contendo esse propágulo não variou com a distância da planta matriz $\left(\chi^{2}=3,0 ; \mathrm{p} \geq 0,05\right)$. Foram observados quatro casos com presença de sementes em meio as fezes de aves dentro de coletores (Figura 2IV).

\subsection{Remoção de frutos}

Do total de 1.000 frutos utilizados no experimento de remoção, 219 (22\%) foram removidos. A média de frutos removidos por parcela foi de $36 \pm 38$ (desvio padrão) no ambiente fechado e $8 \pm 5$ (desvio padrão) no ambiente aberto. Não foi detectada diferença significativa entre as médias de remoção de frutos dos dois ambientes, de acordo com o teste te student ( $\mathrm{p}=0,09: \alpha=0,05)$.

\section{DISCUSSÃO}

O tamanho dos frutos encontrados para Myrceugenia euosma, no presente estudo (4,24 \pm 0,71 $\mathrm{mm})$, permaneceu dentro do esperado pela descrição da espécie (Sobral, 2003), apresentando um tamanho pequeno segundo a classificação realizada por Faustino \& Machado (2006), que considera frutos menores que $10 \mathrm{~mm}$ como de tamanho pequeno. Uma vez que o tamanho determina, em grande parte, se as sementes serão regurgitadas ou defecadas, frutos de menor tamanho (>5 mm), como no caso da M. euosma, são geralmente defecados (Gasperin \& Pizo, 2012).

Tabela 3. Variáveis dos frutos coletados em Myrceugenia euosma, Fazenda das Nascentes, Urupema, SC. $\mathrm{DP}=$ desvio padrão.

Table 3. Fruit variables measured in Myrceugenia euosma fruits, Fazenda das Nascentes, Urupema, SC. DP = Standard deviation.

\begin{tabular}{cccc}
\hline Estatística & Diâmetro $(\mathbf{m m})$ & Peso $(\mathbf{g})^{*}$ & Número de sementes por fruto \\
\hline Média \pm DP & $4,24 \pm 0,71$ & $6,83 \pm 1,33$ & $2,78 \pm 1,56$ \\
\hline Máximo & 5,95 & 8,65 & 8,00 \\
Mínimo & 3,26 & 4,92 & 1,00 \\
n & 57 & 10 & $(50)$ \\
\hline
\end{tabular}

*Peso de lotes com 100 sementes; n: número de unidades amostrais.

Tabela 4. Frequência de coletores $(n=36)$ com propágulos de Myrceugenia euosma localizados em diferentes distâncias da planta matriz, após seis avaliações, na Fazenda das Nascentes, Urupema, SC.

Table 4. Frequency of collectors $(n=36)$ with Myrceugenia euosma propagules, located at different distances from seed tree, after six evaluations, in Fazenda das Nascentes, Urupema, SC.

\begin{tabular}{cccccc} 
Distância & Fruto imaturo & Fruto maduro & Semente & Total \\
$1 \mathrm{~m}$ & 19 & 24 & 16 & 10 & 20 \\
$3 \mathrm{~m}$ & 3 & 7 & 10 & 11 \\
$5 \mathrm{~m}$ & 0 & 32 & 36 & 90 \\
\hline Total & 22 & & 1 & \\
\hline
\end{tabular}


A visitação realizada exclusivamente por aves aos indivíduos com frutos de $M$. euosma sugere que esses animais sejam os principais agentes dispersores da espécie, confirmando o padrão geral descrito para Myrtaceae (Gressler et al., 2006). Outro fator que pode contribuir para aumentar a sua preferência entre as aves é a frutificação em períodos de escassez de outros recursos alimentares (Snow, 1965; Jesus \& Monteiro-Filho, 2007), o que corrobora os resultados encontrados neste estudo: apenas seis espécies arbóreas frutificaram no mesmo período no local de sua realização. Zonotrichia capensis (tico-tico) e Stephanophorus diadematus (cabeça-de-velho) foram as aves com a maior frequência de visitas e de registro de consumo efetivo dos frutos, sem a ocorrência de períodos preferencias para visitação. De maneira geral, as aves apresentam o comportamento de consumir os frutos sem destruir as sementes, mesmo com a passagem pelo trato digestório (Grelle \& Garcia, 1999) que, por sua vez, pode interferir positivamente na germinação de sementes (D’Avila et al., 2010; Guerta et al., 2011). Aves são consideradas excelentes dispersoras pelo fato de depositarem suas fezes em locais distantes das plantas parentais, nos quais as taxas de predação e competição são menores (Francisco \& Galetti, 2001). Isso sugere que $Z$. capensis e $S$. diadematus sejam potenciais dispersores de M. Euosma (Figuras 2I e 2II).

Zonotrichia capensis é classificada na literatura como generalista, apresentando poucas restrições ambientais (Belton, 1994; Sick, 1997) e sendo considerada uma ave residente no ecossistema (Jesus \& Monteiro-Filho, 2007; Pascotto, 2007). Possui hábitos de voos a pequenas distâncias, apresenta comportamento granívoro e, em geral, ao alimentar-se de pequenos frutos os engole por inteiro. Assim, o consumo de frutos de M. euosma pode ser um recurso importante na dieta dessa espécie.

Stephanophorus diadematus pode ser considerada comum entre as espécies típicas de regiões serranas (Sick, 1997; Develey \& Endrigo, 2004), apresentando dieta baseada principalmente em frutos, seguido de artrópodes, flores e folhas (Manhães, 2003; Develey \& Endrigo, 2004; Sazima \& Sazima 2007). É descrita como uma espécie consumidora de polpa, que rasga e despedaça os frutos antes de os comer, descartando as sementes (Montaldo, 2005). No entanto, além desse comportamento, que poderia inviabilizar o processo de dispersão a longas distâncias, foi observado que, em alguns casos, os indivíduos de $S$. diadematus engoliam os frutos de M. euosma por inteiro.

Diversas características de frutos carnosos podem influenciar na atração e na competição por dispersores (Galetti et al., 2011), as principais são cor (Cazetta et al., 2009; Melo et al., 2011), constituintes químicos (Cazetta et al., 2008) e morfologia (Janson, 1983; Levey, 1987; Gasperin \& Pizo, 2012). Espécies que produzem frutos pequenos e em grandes quantidade são classificadas na literatura como generalistas (Howe \& Estabrook, 1977), atraindo uma ampla variedade de espécies de aves, muitas das quais empregam frutos em sua dieta apenas ocasionalmente (Fleming et al., 1987; Francisco \& Galetti, 2002; Athiê \& Dias, 2012). Tal fato poderia explicar o baixo consumo de frutos pelas demais espécies de aves observadas neste estudo. Assim, M. euosma apresenta-se como uma espécie generalista quanto à estratégia de atração de dispersores.

Considera-se que a espécie possui grande potencial para atração da fauna dispersora em geral, principalmente, pelo pequeno tamanho do fruto, fator que contribui para seu consumo sem necessidade de mandibulação por variadas espécies de aves (Athiê \& Dias, 2011). Além disso, a produção de frutos em grande quantidade é uma estratégia para colonizar áreas abertas disponíveis (Howe, 1993), sendo essa característica extremamente importante para processos de ocupação e recomposição de ecossistemas. Nesse contexto, M. euosma pode ser uma espécie potencial para colonização de ambientes alterados.

A frequência de coletores contendo algum propágulo de M. euosma diminuiu com o aumento da distância da planta matriz, corroborando a hipótese da densidade dependente da distância de Janzen-Connell (Janzen, 1970; Howe \& Smallwood, 1982; Francisco \& Galetti, 2001; Swamy et al., 2011). Porém, na comparação com diferentes tipos de propágulos, observaram-se comportamentos distintos entre frutos imaturos, frutos maduros e sementes. Tal fato demonstra que o processo de dispersão próximo da planta-mãe (até $5 \mathrm{~m}$ ) varia em função do propágulo avaliado. Coletores contendo frutos imaturos ou maduros diminuem de frequência em função da distância, isso quer dizer que há maior chance de encontrar esses tipos de propágulos a menor distância da planta matriz. No entanto, coletores contendo sementes não apresentam frequência dependente de distância, configurando-se nesse caso um processo 
aleatório, possivelmente causado pela ação de agentes dispersores.

A presença de coletores com propágulos na distância de $5 \mathrm{~m}$, os quais se encontravam fora da área de projeção da copa de qualquer indivíduo de M. euosma, é um forte indicativo de dispersão zoocórica. Isso fica ainda mais evidente no caso das sementes, que foram encontradas totalmente despolpadas nos coletores. Tal fato, aliado ao registro de algumas sementes em meio a fezes de aves nos coletores, é mais um indício de que a dispersão de $M$. euosma é realizada, principalmente, por aves. Apesar de esses episódios terem sido registrados próximos da planta matriz, aves que apresentam digestão mais lenta podem defecar sementes a distâncias mais longas, apresentando maior eficiência na dispersão (Schupp, 1993; Gasperin \& Pizo 2012). Sendo assim, as aves dispersoras mais importantes são aquelas que engolem frutos inteiros, pois levam todas as sementes contidas no fruto e, possivelmente, as dispersam longe das árvores que as produziram (Moermond \& Denslow, 1985; Marcondes-Machado, 2002).

A taxa de remoção de frutos de $M$. euosma foi relativamente baixa (22\%) e não diferiu entre áreas de mata fechada e aberta. Considera-se que o principal motivo de não terem sido detectadas diferenças entre os ambientes foi a alta variação associada ao número de frutos removidos. Apesar de ter sido um processo que ocorreu em baixa frequência, demonstra ser uma questão importante a ser investigada. Mesmo que não tenha sido possível determinar a causa da remoção dos frutos, a não ocorrência de precipitação durante este experimento sugere que os frutos provavelmente foram removidos pela ação de animais, o que pode caracterizar uma dispersão secundária, ou então uma predação pós-dispersão, conforme observado em outros estudos (Capistrán-Barradas et al., 2006). Embora os autores reconheçam que as informações geradas neste estudo não permitem tais inferências, considera-se que este é um tema relevante e que merece atenção na realização de novos estudos.

\section{CONCLUSÃO}

Os resultados deste estudo indicam que Myrceugenia euosma é uma espécie generalista quanto ao processo de dispersão, com diversos indícios de que as aves são os principais agentes dispersores da espécie. Seus frutos foram consumidos por pelo menos 6 espécies de aves, com predomínio das espécies Stephanophorus diadematus e Zonotrichia capensis. As sementes encontradas intactas em meio a fezes de aves nos coletores são fortes indicativos de ornitocoria na dispersão de Myrceugenia euosma. Ainda, a remoção dos frutos depositados no chão demonstra uma possível ação de outros animais, que podem eventualmente predar as sementes dispersas ou realizar a dispersão secundária. Com isso, fica evidente a necessidade de estudos futuros sobre o tema.

\section{STATUS DA SUBMISSÃO}

Recebido: 29 ago., 2013

Aceito: 26 jun., 2016

\section{AUTOR(ES) PARA CORRESPONDÊNCIA}

\section{Newton Clóvis Freitas da Costa}

Departamento de Engenharia Florestal, Universidade do Estado de Santa Catarina UDESC, Avenida Luiz de Camões, 2090, Conta Dinheiro, CEP 88520-000, Lages, SC, Brasil e-mail: newtoncfc@gmail.com

\section{REFERÊNCIAS}

Alvares CA, Stape JL, Sentelhas PC, Gonçalves JLM, Sparovek G. Köppen's climate classification map for Brazil. Meteorologische Zeitschrift 2014; 22(6): 711-728. http:// dx.doi.org/10.1127/0941-2948/2013/0507.

Athiê S, Dias MM. Frugivoria e dispersão de sementes por aves em Casearia sylvestris Sw. (Salicaceae) na região centro-leste do Estado de São Paulo. Revista Brasileira de Zoociências 2011; 13(1-3): 79-86.

Athiê S, Dias MM. Frugivoria por aves em um mosaico de Floresta Estacional Semidecidual e reflorestamento misto em Rio Claro, São Paulo, Brasil. Acta Botanica Brasílica 2012; 26(1): 84-93. http://dx.doi.org/10.1590/ S0102-33062012000100010.

Belton W. Aves do Rio Grande do Sul: distribuição e biologia. São Leopoldo: Unisinos; 1994.

Capistrán-Barradas A, Moreno-Casasola P, Defeo O. Post dispersal fruit and seed removal by the crab Gecarcinus lateralis in a coastal forest in Veracruz, México. Biotropica 2006; 38(2): 203-209. http://dx.doi.org/10.1111/j.17447429.2006.00116.x.

Cazetta E, Schaefer H, Galetti M. Why are fruits colorful? The relative importance of achromatic and chromatic 
contrasts for detection by birds. Evolutionary Ecology 2009; 23(2): 233-244. http://dx.doi.org/10.1007/s10682007-9217-1.

Cazetta E, Schaefer MH, Galetti M. Does attraction to frugivores or defense against pathogens shape fruit pulp composition? Oecologia 2008; 155(2): 277-286. PMid:18043946. http://dx.doi.org/10.1007/s00442-0070917-6.

Cordeiro N, Howe HF. Forest fragmentation severs mutualism between seed dispersers and an endemic African tree. Proceedings of the National Academy of Sciences of the United States of America 2003; 100(24): 14052-14056. PMid:14614145. http://dx.doi.org/10.1073/ pnas.2331023100.

Côrtes MC, Cazetta E, Staggemeier VG, Galetti M. Linking frugivore activity to early recruitment of a bird dispersed tree, Eugenia umbelliflora (Myrtaceae) in the Atlantic rainforest. Austral Ecology 2009; 34(3): 249-258. http:// dx.doi.org/10.1111/j.1442-9993.2009.01926.x.

D’Avila G, Gomes-Jr A, Canary AC, Bugoni L. The role of avian frugivores on germination and potential seed dispersal of the Brazilian Pepper Schinus terebinthifolius. Biota Neotropica 2010; 10(3): 45-51. http://dx.doi. org/10.1590/S1676-06032010000300004.

Develey PF, Endrigo E. Aves da Grande São Paulo, guia de campo. São Paulo: Aves e Fotos Editora; 2004.

Empresa Brasileira de Pesquisa Agropecuária - EMBRAPA. Sistema Brasileiro de Classificação de solos. Rio de Janeiro: Centro Nacional de Pesquisas de Solos; 1999.

Faustino TC, Machado CG. Frugivoria por aves em uma área de campo rupestre na Chapada Diamantina, BA. Revista Brasileira de Ornitologia 2006; 4(2): 137-143.

Fleming TH, Breitwisch R, Whitesides GH. Patterns of tropical vertebrate frugivore diversity. Annual Review of Ecology and Systematics 1987; 18(1): 91-109. http://dx.doi. org/10.1146/annurev.es.18.110187.000515.

Forget PM. Post-dispersal predation and scatterhoarding of Dipteryx panamensis (Papilionaceae) seeds by rodents in Panama. Oecologia 1993; 94(2): 255-261. PMid:28314040. http://dx.doi.org/10.1007/BF00341325.

Francisco MR, Galetti M. Frugivoria e dispersão de sementes em Rapanea lancifolia (Myrsinaceae) por aves numa área de cerrado do Estado de São Paulo, sudeste do Brasil. Ararajuba 2001; 9: 13-19.

Francisco MR, Galetti M. Aves como potenciais dispersoras de sementes de Ocotea pulchella Mart. (Lauraceae) numa área de vegetação de cerrado do sudeste brasileiro. Revista Brasileira de Botanica. Brazilian Journal of Botany 2002; 25(1): 11-17. http://dx.doi.org/10.1590/S010084042002000100003.

Frankie GW, Baker HG, Opler PA. Comparative phenological studies of trees in tropical wet and dry forests in the lowlands of Costa Rica. Journal of Ecology 1974; 62(3): 881-913. http://dx.doi.org/10.2307/2258961.

Galetti M, Pizo MA, Morellato LPC. Fenologia, frugivoria e dispersão de sementes. In: Cullen JRL, Rudran R, Valladares-Padua C. Métodos de estudos em biologia da conservação e manejo da vida silvestre. 2. ed. Curitiba: UFPR; 2006.

Galetti M, Pizo MA, Morellato LPC. Diversity of functional traits of fleshy fruits in a species-rich Atlantic rain forest. Biota Neotropica 2011; 11(1): 181-193. http://dx.doi. org/10.1590/S1676-06032011000100019.

Gasperin G, Pizo MA. Passage time of seeds through the guts of frugivorous birds, a first assessment in Brazil. Revista Brasileira de Ornitologia 2012; 20(1): 48-51.

Grelle CEV, Garcia QS. Potential dispersal of Cecropia hololeuca by the commom opossum (Didelphis aurita) in Atlantic Forest, southastern Brazil. Revue ecologie. La Terre et la Vie 1999; 54: 327-332.

Gressler E, Pizo MA, Morellato LPC. Polinização e dispersão de sementes em Myrtaceae do Brasil. Revista Brasileira de Botanica. Brazilian Journal of Botany 2006; 29(4): 509-530. http://dx.doi.org/10.1590/S0100-84042006000400002.

Guerta RS, Lucon LG, Motta-Junior JC, Vasconcellos LAS, Figueiredo RA. Bird frugivory and seed germination of Myrsine umbellata and Myrsine lancifolia (Myrsinaceae) seeds in a cerrado fragment in southeastern Brazil. Biota Neotropica 2011; 11(4): 59-65. http://dx.doi.org/10.1590/ S1676-06032011000400005.

Hansen DM, Galetti M. The forgotten megafauna. Science 2009; 324(5923): 42-43. PMid:19342573. http://dx.doi. org/10.1126/science.1172393.

Harper JL. Population biology of plants. London: Academic Press; 1977.

Howe HF, Estabrook GF. On intraspecific competition for avian dispersers in tropical trees. American Naturalist 1977; 111(981): 817-832. http://dx.doi.org/10.1086/283216.

Howe HF, Miriti MN. When seed dispersal matters. Bioscience 2004; 54(7): 651-660. http://dx.doi.org/10.1641/00063568(2004)054[0651:WSDM]2.0.CO;2.

Howe HF, Smallwood J. Ecology of seed dispersal. Annual Review of Ecology and Systematics 1982; 13(1): 201-228. http://dx.doi.org/10.1146/annurev.es.13.110182.001221.

Howe HF. Aspects of variation in a Neotropical seed dispersal system. Vegetation 1993; 107(108): 149-162.

Janson CH. Adaptation of fruit morphology to dispersal agents in a neotropical forest. Science 1983; 219(4581): 187-189. PMid:17841688. http://dx.doi.org/10.1126/ science.219.4581.187.

Janzen DH. Herbivories and the number of tree species in tropical forests. American Naturalist 1970; 104(940): 501-528. http://dx.doi.org/10.1086/282687. 
Jesus S, Monteiro-Filho ELA. Frugivoria por aves em Schinus terebinthifolius (Anacardiaceae) e Myrsine coriacea (Myrsinaceae). Revista Brasileira de Ornitologia 2007; 15(4): 585-591.

Jordano P, Godoy JA. Frugivore-generated seed shadows: a landscape view of demographic and genetic effects. In: Levey DJ, Silva WR, Galleti M. Seed dispersal and frugivory: ecology, evolution and conservation. New York: CABI Publishing; 2002.

Legrand CD, Klein R. Myrtaceae. In: Reitz P. Flora Ilustrada de Santa Catarina. Itajaí: Herbário Barbosa Rodrigues; 1970.

Levey DJ. Seed size and fruit-handling techniques of avian frugivores. American Naturalist 1987; 129(4): 471-485. http://dx.doi.org/10.1086/284652.

Liebsch D. Síndromes de dispersão de diásporos de um fragmento de floresta ombrófila mista em Tijucas do Sul, PR. Revista Acadêmica: Ciência Animal 2007; 5(2):167-175.

Manhães MA. Variação sazonal da dieta e do comportamento alimentar de Traupíneos (Passeriformes: Emberizidae) em Ibitipoca, Minas Gerais, Brasil. Ararajuba 2003; 11(1): 45-55.

Marcondes-Machado LO. Comportamento alimentar de aves em Miconia rubiginosa (Melastomataceae) em fragmento de Cerrado. Iheringia. Série Zoologia 2002; 92(3): 97-100. http://dx.doi.org/10.1590/S0073-47212002000300010.

Martins-Ramos D, Chaves CL, Bortoluzzi RLC, Mantovani A. Florística de Floresta Ombrófila Mista Altomontana e de Campos em Urupema, Santa Catarina, Brasil. Revista Brasileira de Biociências 2011; 9(2): 156-166.

Mauhs J, Backes A. Estrutura fitossociológica e regeneração natural de um fragmento de Floresta Ombrófila Mista exposto a perturbações antrópicas. Pesquisas Botânica 2002; 52(4): 89-109.

Melo GL, Penatti NC, Raizer J. Fruit of contrasting colour is more detectable by frugivores. Journal of Tropical Ecology 2011; 27(3): 319-322. http://dx.doi.org/10.1017/ S0266467410000775.

Moermond TC, Denslow JS. Neotropical avian frugivores: patterns of behavior, morphology and nutrition, with consequences for fruit selection. Ornithological Monographis 1985; 36(36): 865-897. http://dx.doi.org/10.2307/40168322.

Montaldo NH. Aves frugívoras de un relicto de selva subtropical ribereña en Argentina: manipulación de frutos y destino de las semillas. El Hornero 2005; 20(2): 163-172.

Pascotto MC. Rapanea ferruginea (Ruiz \& Pav.) Mez. (Myrsinaceae) como uma importante fonte alimentar para as aves em uma mata de galeria no interior do estado de São Paulo. Revista Brasileira de Zoologia 2007; 24(3): 735774. http://dx.doi.org/10.1590/S0101-81752007000300026.

Pizo MA. Padrão de deposição de sementes e sobrevivência de sementes e plântulas de duas espécies de Myrtaceae na Mata Atlântica. Revista Brasileira de Botanica 2003;
26(3): 371-377. http://dx.doi.org/10.1590/S010084042003000300010 .

Pizo MA. Frugivory and habitat use by fruit-eating birds in a fragmented landscape of southeastern Brazil. Ornitologia Neotropical 2004; 15: 117-126.

Saravy FP, Freitas PJ, Lage MA, Leite SJ, Braga LF, Sousa MP. Síndrome de dispersão em estratos arbóreos em um fragmento de Floresta Ombrófila Aberta e Densa em Alta Floresta- MT. Alta Floresta 2006; 2(1): 1-12.

Sazima I, Sazima M. Petiscos florais: pétalas de Acca sellowiana (Myrtaceae) como fonte alimentar para aves em área urbana no Sul do Brasil. Biota Neotropica 2007; 7(2): 307-312. http://dx.doi.org/10.1590/S167606032007000200035 .

Schupp EW. Quantity, quality and the effectiveness of seed dispersal. Vegetatio 1993; 107(1): 15-29.

Sick H. Ornitologia Brasileira. 2. ed. Rio de Janeiro: Nova Fronteira, 1997.

Silva JCB, Cândido JF Jr, Vogel HF, Campos JB. Dispersão por aves de Psidium guajava L. (Myrtaceae) em ambiente ripário na bacia do rio Paraná, Brasil. Semina. Ciências Biológicas e da Saúde 2013; 34(2): 195-204. http://dx.doi. org/10.5433/1679-0367.2013v34n2p195.

Silva JMC, Tabarelli M. Tree species impoverishment and the future flora of the Atlantic forest of northeast Brazil. Nature 2000; 404(6773): 72-74. PMid:10716443. http:// dx.doi.org/10.1038/35003563.

Snow DW. A possible selective factor in the evolution of fruiting seasons in tropical forest. Oikos 1965; 15(2): 274-281. http://dx.doi.org/10.2307/3565124

Sobral M. A família Myrtaceae no Rio Grande do Sul. São Leopoldo: Ed. Unisinos; 2003.

Sonego R, Backes A, Souza A. Descrição da estrutura de uma Floresta Ombrófila Mista, RS, Brasil, utilizando estimadores não-paramétricos de riqueza e rarefação de amostras. Acta Botanica Brasílica 2007; 21(4): 943-955. http://dx.doi.org/10.1590/S0102-33062007000400019.

Swamy V, Terborgh J, Dexter KG, Best BD, Alvarez P, Cornejo F. Are all seeds equal? Spatially explicit comparisons of seed fall and sapling recruitment in tropical forest. Ecology Letters 2011; 14(2): 195-201. PMid:21176051. http://dx.doi.org/10.1111/j.1461-0248.2010.01571.x.

Terborgh J. Community aspects of frugivory in tropical Forest. In: Estrada A, Fleming TH. Frugivores and seed dispersal. Dordrech: Dr. W. Junk Publishers; 1986.

Zar JH. Biostatistical analysis. 2. ed. New Jersey: PrenticeHall; 1984. 